\title{
El uso de las TIC en los sistemas educativos formales. Propuesta de marco metodológico para su caracterización y análisis
}

\section{The use of ICTs in formal education systems. Proposal for a methodological framework for characterization and analysis}

MARTÍNEZ-MÁRQUEZ, Marco Antonio †*, CASILLAS-MARTÍNEZ, María Elena, HUIZARRUVALCABA, Diego y MOTA-MACÍAS, Silvia Elena

Centro Universitario del Norte de la Universidad de Guadalajara, Kilómetro 191, Carretera Federal Número 23, Código Postal 46200, Colotlán, Jalisco, México

ID $1^{\mathrm{er}}$ Autor: Marco Antonio, Martínez-Márquez / ORC ID: 0000-0003-2898-6687, Researcher ID Thomson: S-7641-2018, PubMed Autor ID: 6389e65a779dbab5d5a321412226c3915f08, CVU CONACYT ID: 825933

ID $1^{\mathrm{er}}$ Coautor: María Elena, Casillas-Martínez / ORC ID: 0000-0002-3403-0350, Researcher ID Thomson: X-3125-2018, CVU CONACYT ID: 953359

ID $2^{\text {do }}$ Coautor: Diego, Huizar-Ruvalcaba / ORC ID: 0000-0001-7063-5579, Researcher ID Thomson: X-2941-2018, PubMed Autor ID: 33040dc75ac995545d8e1bc86d3536252008, CVU CONACYT ID: 953304

ID $3^{\text {er }}$ Coautor: Silvia Elena, Mota-Macías / ORC ID: 0000-0002-0098-4295, Researcher ID Thomson: X-3109-2018, PubMed Autor ID: ebf4bdf1b141bbf6b36b6972e40892ce1208, CVU CONACYT ID: 953357

\section{Resumen}

Es indudable que fue el surgimiento de las tecnologías de la información y la comunicación (TIC) lo que revolucionó la época actual, dando un giro radical a la forma en que nos comunicamos, aprendemos y trabajamos, situación que hizo considerar a la informacion el recurso más valiso para el progreso, y la educación el medio para producirlo y distribuirlo, lo anterior llevó a los gobiernos a generar altas expectativas respecto al uso de las tecnologías en la educación, al considerar que ofrecían la posibilidad de innovar los procesos de enseñanza y mejorar los de aprendizaje, siendo motivo de declaración mundial de la UNESCO; sin embargo, estudios recientes demuestran que no ha sido fácil la incorporación y uso de las TIC en las aulas por diversos motivos, destacando el costo económico que representan, el reto de la alfabetización digital de docentes y alumnos, y lo más importante, el uso que éstos hacen de ellas. En este sentido, en el presente trabajo se propone un marco metodológico, entendido como el conjunto de acciones orientadas a describir y analizar un objeto de investigación, en este caso el uso de las tecnologías en la educación formal, para lo cual se realiza un minucioso recorrido para la elección del paradigma, perspectiva humanístico-cualitativa, método, orientación, alcance del estudio, técnicas de recolección y análisis de datos, así como la determinación de la población y muestra.

Educación, TIC, Uso, Caracterización, Análisis, Marco metodológico

\section{Abstract}

Undoubtably, the rise of technology of the information and communication (ITCs) was what revolutionized the current epoch, giving a radical turn to the way in which we communicate, learn, and work-a situation that led to considering information the most valuable resource toward progress and education the médium through which to produce it and distribute it. This led governments to generate high expectations regarding the use of technologies in education, believing that they offered the possibility of innovating teaching processes and improve learning processes, it being a motive for a UNESCO world declaration. However, recent studies demonstrate that the incorporation and use of ITCs in classrooms have not been easy for various reasons, especially the financial cost they represent, the challenge of digital literacy for teachers and students, and, most importantly, the use that they make of ITCs. In this regard, in this work, a methodological framework is proposed, understood as a set of actions geared toward describing and analyzing an object of research orientadas, in this case, the use of technologies in formal education. For this purpose, a meticulous survey is performed to choose a paradigm, a qualitative-humanistic perspective, a method, an orientation, the scope of the study, and techniques for data gathering and análisis, as well as to determine the population and sample.

Education, ITCs, Use, Characterization, Analysis, Methodological framework

Citación: MARTÍNEZ-MÁRQUEZ, Marco Antonio, CASILLAS-MARTÍNEZ, María Elena, HUIZAR-RUVALCABA, Diego y MOTA-MACÍAS, Silvia Elena. El uso de las TIC en los sistemas educativos formales. Propuesta de marco metodológico para su caracterización y análisis. Revista Teoría Educativa. 2019. 3-7: 21-33.

\footnotetext{
* Correspondencia del Autor (correo electrónico: licmarco@ cunorte.udg.mx)

$\dagger$ Investigador contribuyendo como primer autor.
} 


\section{Introducción}

Partiendo de la premisa de que el uso de las TIC está cada vez más generalizado en todos los ámbitos sociales, y que el educativo no es la excepción, al considerar que con su incorporación a las aulas se puede innovar los procesos de enseñanza y mejorar de los de aprendizaje, pero siendo consientes que los resultados de estudios recientes muestran resultados contradictorios, además de evidenciar las mútiples dificultades que ésto ha implicado, y que el éxito que se tenga depende del trabajo específico que se realice en cada contexto, es que se justifica la caracterización y análisis de casos particulares, que permitan la generación e implementación de proyectos de intervención para mejorar el uso de las TIC en dichos procesos de enseñanzaaprendizaje.

En este orden de ideas, en el presente trabajo se hace la propuesta de un marco metodológico que ayude precisamente en la caracterización de esos casos particulares, para lo cual se parte de los conceptos básicos de ciencia, investigación, conocimiento, método, observación, experimentación e investigación educativa, para después proporcionar los elementos necesarios para la adecuada elección de un paradigma que permita describir preguntas de investigación en el proceso de interpretación y la realización de descripciones detalladas, una perspectiva humanística que facilite el abordaje de actividades prácticas y razonamientos sociológicos, un método que promueva el análisis de casos particulares para extraer conclusiones generales, una orientación que se interese por lo que hacen las personas, apoyándose en técnicas de recolección de datos como: la observación, notas, entrevistas, grabaciones y registros, y en técnicas de análisis como: la categorización, saturación y triangulación, el alcance del estudio dirigido a responder por las causas de los fenómenos; y, finalmente, para la elección de la población y muestra, que garantice objetividad en la información, y que será la base primero para la caracterización pretendida y segundo para la elaboración de un proyecto de intervención.

\section{Conceptos básicos}

"Se puede llegar a conocer la naturaleza de los fenómenos a través de la experiencia, el razonamiento y la investigación. Estas tres vías no son mutuamente excluyentes, sino más bien complementarias" (Cohen y Manion; 1986, tomado de Bisquerra; 1989: 1).
Antes de presentar una propuesta de marco metodológico, entendido como el conjunto de acciones encaminadas a describir y analizar un objeto de investigación, en este caso el uso de las TIC en la educación, se hace indispensable referirse a la naturaleza de la investigación científica, por medio de algunos conceptos básicos que ayudarán en la elección del paradigma, la perspectiva, el método, la orientación, los alcances del estudio, y las técnicas de recolección y análisis de datos.

El primer concepto para destacar es el de ciencia, definida como "... un conjunto organizado de conocimientos que han sido adquiridos mediante el método científico. La preocupación por la explicación de los fenómenos es lo que ha dado lugar a la ciencia" (Bisquerra; 1989:2).

A la definición presentada, agrega el autor que existen dos tendencias sobre el objetivo de la ciencia: para unos es contribuir al conocimiento teórico, aquí se encuentra, por ejemplo, la explicación de los fenómenos naturales, y se incluye a los que proponen una metodología científica clásica apegada al positivismo, según esta concepción la ciencia es nomotética, está dirigida al establecimiento de leyes generales y utiliza principalmente métodos cuantitativos; otros en cambio, se inclinan por las corrientes humanistas, que promueven una metodología cualitativa, privilegiando la profundidad y comprensión de lo investigado, aquí se encuentra, por ejemplo, la investigación acción, donde se afirma que la función de la ciencia es comprender para transformar; desde este punto de vista la ciencia es idiográfica, y se basa en el conocimiento individual.

Bajo estas premisas, una investigación basada en describir, analizar e interpretar la forma en que docentes y alumnos hacen uso de las tecnologías, coincide con la segunda tendencia, pues no tiene como fin el establecimiento de leyes generales, por el contrario, se pretende conocer aspectos particulares, para dictar lineamientos que permitan propiciar un cambio en beneficio de los alumnos, lo que requiere profundidad y comprensión de lo investigado, así como el empleo de una metodología cualitativa. 
Pero si hablar de ciencia es hacerlo de conocimientos organizados, adquiridos a través del método científico, con el objetivo de explicar fenómenos particulares; y, para llegar a conocer la naturaleza de éstos, los caminos posibles son, como se señala en la cita inicial, la experiencia, que opera en el ámbito de los acontecimientos cotidianos; el razonamiento, que puede ser de tres tipos, deductivo bajo las ideas de Aristóteles, inductivo según el pensamiento de Francisco Bacón o hipotético deductivo si se conjunta los anteriores; $y$, la investigación, como actividad que combina la experiencia y el razonamiento, teniendo como característica la utilización del método científico, el segundo concepto básico a destacar es el de investigación científica, definida como: “... una investigación sistemática, controlada, empírica y crítica, de proposiciones hipotéticas sobre supuestas relaciones que existen entre fenómenos naturales" (kerlinger; 1985: 7, tomado de Bisquerra; 1989: 1)

Ahora bien, lo dicho en el párrafo que antecede, obliga a reflexionar sobre la importancia de la experiencia que debe poseer el investigador para aproximarse a la realidad objeto de estudio, y el tipo de razonamiento a realizar para obtener una idea profunda del fenómeno investigado; eso ayudará más adelante en la elección del método, la orientación de la investigación, e incluso las técnicas de recolección y análisis de datos, con lo cual se estará es la posibilidad de generar conocimiento científico, que exige del investigador un pensamiento dinámico, crítico y con apertura a la realidad.

Acorde a lo presentado, el tercer concepto relevante es el de conocimiento científico, que según Bisquerra (1989: 3): “... es el producto que se obtiene mediante la aplicación del método científico", teniendo como algunas de sus características: origen empírico, método, análisis, especialización, duda metódica, incertidumbre, probabilístico, medición, precisión, profundidad, objetividad, comprobabilidad, relacionabilidad, sistemático, explicativo y predictivo, además de tender a mejorar las condiciones de vida, impulsar el progreso y ser útil.
Como se puede ver, un concepto lleva a otro, y el de conocimiento científico conduce ahora al de método científico, definido como “... un proceso sistemático por medio del cual se obtiene el conocimiento científico basándose en la observación y la experimentación" (Bisquerra; 1989: 5); en ese tenor, la importancia del método radica en que está destinado a explicar fenómenos, establecer relaciones entre los hechos que se observan y enunciar leyes que expliquen esos fenómenos, y le permitan, con los conocimientos obtenidos, generar aplicaciones útiles. Agrega el autor que para su implementación es preciso mantener una forma planificada de trabajar, pues los conocimientos que se generen sirven de base a investigaciones futuras.

Pero cada paso del método científico permite al investigador conocer a profundidad su objeto de estudio, y presentar resultados ante la comunidad del conocimiento, por ello la base de la investigación se centra en otros conceptos relevantes que debemos tratar previo a la definición del marco metodológico y que son la observación y la experimentación, considerados como:

.. procesos esenciales del método científico. La observación considera los fenómenos tal como se presentan, sin modificarlos, ni actuar sobre ellos. La experimentación implica una manipulación intencionada por parte del experimentador sobre las condiciones en que se desarrolla el fenómeno. El observador escucha la naturaleza, el experimentador la interroga. La preponderancia de una sobre la otra da lugar a distintos métodos de investigación. (Bisquerra; 1989: 7).

De acuerdo con el autor de la cita, la observación permite al investigador explorar hechos y fenómenos que pueden ser percibidos por los sentidos; incide en la curiosidad que se posee de conocer la naturaleza, pues lo primero que se hace al encontrar un fenómeno interesante es observarlo con atención. En cambio, la experimentación busca que el investigador compruebe si su hipótesis es cierta o no, consiste en reproducir y observar varias veces el fenómeno, modificando las circunstancias que se consideren pertinentes. 
Con base en las posibilidades presentadas, es preciso señalar que no todas las investigaciones tienen la misma intención y alcance, por lo que la experimentación aparece cuando el estudio y su diseño metodológico lo requieren.

Finalmente, previo a definir el marco metodológico a utilizar, es importante abordar el concepto de investigación educativa, considerada por Bisquerra (1989: 8) como: "un conjunto sistemático de conocimientos acerca de la metodología científica aplicada a la investigación educativa de carácter empírico".

En el mismo sentido, McMillan (2005: 4), señala que: "La Investigación Educativa es un estudio científico y sistemático que utiliza aproximaciones cualitativas y cuantitativas"; agrega el autor que se trata de un tipo de investigación que puede ser básica, aplicada o evaluativa, y cuya importancia radica en las siguientes razones: primero, que los docentes constantemente tratan de comprender los procesos educativos respecto de los cuales tiene que tomar decisiones; segundo, que grupos de poder no educativos, y el propio Estado, han introducido cambios en los sistemas educativos; tercero, que en los últimos años se han incrementado las actividades de investigación en el ámbito educativo; cuarto, que se han incrementado también las revisiones sobre las investigaciones previas, lográndose nuevas interpretaciones sobre la evidencia empírica acumulada; quinto, que la investigación educativa es un tópico de fácil acceso; y, sexto, que muchos investigadores de tiempo parcial consultan el cúmulo de trabajos en educación para realizar otros estudios.

Como se puede ver, abordar el concepto y características de la investigación educativa cobra relevancia, dado que es aquí donde se circunscribe la propuesta que en adelante se plantea; es decir, el conocimiento acerca de la metodología aplicada a este tipo de estudios, será el que contribuya para describir, analizar e interpretar lo que está sucediendo en un contexto con relación al uso de las tecnologías de la información y la comunicación, así como para buscar alternativas para el mejoramiento de los procesos formativos en beneficio de los alumnos.
Desarrollados los conceptos básicos, y de acuerdo con las necesidades particulares de este tipo de investigaciones, la propuesta de marco metodológico es la siguiente.

\section{El paradigma}

"Los paradigmas son realizaciones científicas universalmente reconocidas, que, durante cierto tiempo proporcionan modelos de problemas y soluciones a una comunidad científica" (Bisquerra; 1989: 45).

El primer aspecto metodológico que se debe definir en una investigación es el paradigma a utilizar; en este sentido, y sin restar la importancia que tiene el paradigma cuantitativo, se elige el cualitativo, ya que el interés en este tipo de trabajos no es numérico, ni de establecimiento de leyes, sino de descripciones y cualidades, y este último "Utiliza la recolección de datos sin medición numérica para descubrir o afinar preguntas de investigación en el proceso de interpretación" (Hernández; 2010: 7). Se trata de un paradigma de investigación que: “... surge como alternativa al paradigma racionalista, puesto que en las disciplinas del ámbito social existen diferentes problemáticas... que no se pueden explicar en toda su extensión desde la metodología cuantitativa" (Pérez; 1994: 26).

Así, ante la necesidad de caracterizar las prácticas en uso de las TIC por parte de los docentes, con la finalidad de tener un panorama general de la situación que guarda el fenómeno, para contrastarlo con la teoría, se trata de una cuestión que sólo con el paradigma elegido será posible, pues se busca recuperar datos cualitativos para analizarlos e interpretarlos, los que son definidos por Hernández (2010: 9) como: "Descripciones detalladas de situaciones, eventos, personas, interacciones, conductas observadas y sus manifestaciones" Más aún, son datos que tienen un patrón cultural, considerado como el "Común denominador de los marcos de interpretación cualitativos, que parte de la premisa de que toda cultura o sistema social tiene un modo único para entender situaciones y eventos" (Hernández; 2010: 10). Lo anterior sólo es posible desde el paradigma cualitativo y requiere, del investigador, desprenderse $\mathrm{y}$ alejarse del análisis, para que las aseveraciones que surjan sean apegadas a la realidad. 
Asimismo, dentro de las características que posee el paradigma cualitativo y que favorecen al tipo de estudio, se encuentran, según Hernández (2010), la exploración de fenómenos en profundidad, donde el investigador indaga minuciosamente la situación; se conduce en ambientes naturales, como en este caso donde la recolección de información se lleva a cabo dentro de la institución, y de manera directa con los actores involucrados en el uso de las tecnologías; los significados se extraen de los datos, una respuesta por parte del docente da mayor énfasis que un dato numérico; $y$, no se fundamenta en la estadística, por el contrario, lo hace en los aspectos finos aportados por los sujetos.

En conclusión, el proceso que se lleva con el acompañamiento del paradigma cualitativo, es inductivo, parte de los datos recabados, es recurrente, analiza las realidades subjetivas que emergen a lo largo de la investigación, y sobre todo, posee flexibilidad, ya que no tiene una secuencia lineal a la cual deba sujetarse el investigador, sino que ha de construir y reconstruir, avanzando y retrocediendo dentro del proceso cuantas veces sea necesario.

Las bondades del paradigma son la profundidad de significados que se obtienen del análisis de los datos, la amplitud que posibilita abarcar toda la información obtenida, la riqueza interpretativa y la contextualización del fenómeno. Por todo esto, se sostiene la elección del paradigma cualitativo.

\section{La perspectiva}

"Dentro de la reacción al positivismo surge el existencialismo y la metodología cualitativa. En esta línea surge la psicología humanista de Carl Rogers, que estudia al individuo, no el grupo; pero, el anti-positivismo en investigación en Ciencias Sociales, está representado básicamente por la fenomenología, la etnometodología y el interaccionismo simbólico" (Bisquerra; 1989: 59).
La elección de la perspectiva dentro del paradigma, es una actividad que se encuentra directamente relacionada con lo que se pretende investigar, el grado de profundidad necesario, la información requerida, las condiciones particulares de quienes generan los hechos objeto de estudio y del investigador, y con el tiempo y el espacio; así, en una investigación sobre uso de las TIC en educación, por sus características, la perspectiva propuesta es la etnometodológica, que permite, según el autor citado, dos posibilidades de acción, de un lado, asumir una posición externa para observar y escuchar, y del otro, participar en conversaciones para captar los significados que los sujetos asignan a sus conductas; ésta última más acorde a lo que se busca, y que es saber el porqué de ciertas formas de utilizar las tecnologías de la información y la comunicación por parte de los profesores y alumnos.

Sobre la perspectiva considerada agrega Bisquerra (1989), que el término etnometodología fue acuñado por Harold Garfinkel para referirse a una corriente que pretende ser una reflexión sobre la construcción del saber social; que es una orientación que busca captar la complejidad de las prácticas discursivas de los individuos en contextos sociales; que se focaliza en la utilización del lenguaje en la práctica social y trata de analizar cómo las personas mantienen un sentido de la realidad, lo que hace suponer que la cultura de la institución juega un papel preponderante, en la forma como los sujetos adoptan los cambios que se les plantean en la manera de llevar a cabo sus labores. La etnometodología trata entonces, “... de actividades prácticas, de circunstancias prácticas y razonamientos sociológicos prácticos... En lugar de estudiar acontecimientos extraordinarios, se dedica a las actividades de la vida cotidiana. Analiza cómo la gente da sentido a su mundo cotidiano..." (Bisquerra; 1989: 59).

De acuerdo con el autor citado, el investigador accede a la opinión de los participantes respecto de los fenómenos que se investigan, se propone que los hechos sociales no sean considerados como objetos, sino como realizaciones prácticas; de ahí que el principal rasgo que los distingue es el reconocimiento que se hace del carácter contextual de todo hecho social. 
En el mismo sentido de las ideas anteriores, e insistiendo en la elección de la perspectiva etnometodológica, se puede particularizar que obedece al tipo de información que permite obtener en el desarrollo de un trabajo de investigación, y que en palabras de Fuentes (1990), son cuestiones como la forma en que las personas organizan su vida diaria en sociedad, y de cómo hacen que sus actividades sean significativas, en este caso, de cómo los profesores organizan su actividad docente, mediante el uso de las TIC, haciendo que sea significativo, sobre todo, para los alumnos como receptores de dicha actividad. Lo anterior se encuentra directamente relacionado con el hecho de que, como disciplina:

Procura comprender la situación social "desde adentro", tal como aparece a los hombres que la viven; trata de transmitir el sentido que tienen ellos de las cosas, evitando en especial las conceptualizaciones convencionales de la sociología normal; lo que no significa que no esté influido por ellas. (Fuentes; 1990: 115).

Así, para la autora citada, la etnometodología, se encuentra influenciada, principalmente, por la fenomenología y la filosofía del lenguaje ordinario, lo que la orienta al estudio de lo cotidiano, en oposición lo científico; el sentido común, como depósito de ideas y prácticas; y, la conciencia colectiva, con atención en la estructura de las reglas y el conocimiento compartido, que permiten una interacción social estable. Finalmente reitera, que según Garfinkel "lo que cohesiona el mundo social no es una moralidad con un matiz sagrado, sino una densa estructura colectiva de entendimientos tácitos... referentes a los asuntos mundanos y triviales entendimientos a los cuales... no suele atribuirse ninguna importancia especial" (Fuentes; 1990: 116). Con lo anterior se demuestra la importancia que tiene el conocimiento del contexto por parte del investigador, pues se trata del lugar y tiempo donde se desarrollan los hechos cotidianos que investiga, y que influye sobre las conductas de los sujetos involucrados, del que se obtienen los datos a analizar; así, la perspectiva etnometodológica facilitará que el investigador se interne en el espacio natural, conviva, entreviste y recupere información, que le dé evidencia de esas interacciones sociales, y verificar si una pregunta de investigación es cierta y se puede demostrar, o simplemente caracterizar la situación.

\section{El método}

"Un método es el camino para llegar a un fin. Los métodos de investigación constituyen el camino para llegar al conocimiento científico, son un procedimiento o conjunto de procedimientos que sirven de instrumento para alcanzar los fines de la investigación" (Bisquerra; 1989: 55).

Definido el empleo de la metodología cualitativa que permite descripciones detalladas e identificación de cualidades, y la perspectiva etnometodológica que facilita el estudio de actividades prácticas, corresponde decidir el método, en tanto conjunto de procedimientos lógicos a través de los cuales se plantean los problemas científicos y se pone a prueba las hipótesis y los instrumentos de trabajo, para demostrar si un argumento es válido o no; al respecto, y como quedó establecido en la primera parte de este documento, son tres las posibilidades que existen, el método deductivo, el inductivo, y el hipotético deductivo si se hace una combinación de los dos primeros.

En este trabajo y teniendo presente los fines que se persiguen, se recomienda el método inductivo, ya que posibilita el análisis de hechos particulares para extraer conclusiones de carácter general, y su objetivo es el descubrimiento de generalizaciones a partir de observaciones sistemáticas de la realidad; sobre el mismo, afirma Bisquerra (1989), que el trabajo inicia con una recolección de datos tomados en campo y de los sujetos involucrados, se categorizan las variables observadas, se establecen regularidades $\mathrm{y}$, al final, relaciones entre dichos datos.

\section{La orientación}

"Entre las principales modalidades del método inductivo están los estudios descriptivos, correlacionales, la orientación etnográfica y la investigación acción" (Bisquerra; 1989: 62).

A partir del objetivo pretendido, identificado como caracterizar las prácticas en uso de las TIC por parte de los docentes, y en su caso proponer lineamientos para el mejoramiento de las mismas, es la orientación etnográfica la que mejor ayuda para su logro, pues cuando se habla de etnografía, lo que se está haciendo es referirse a un término derivado de la Antropología que significa: 
"Descripción del modo de vida de una raza o grupo de individuos" (Bisquerra; 1989: 146); la etnografía, siendo descriptiva por definición, contribuye para conocer, describir e interpretar (caracterizar), lo que un grupo de personas, en este caso los profesores, están haciendo con relación al fenómeno investigado, aquí el uso de las tecnologías, además, se trata de un enfoque de investigación cualitativa. Agrega el autor, que la etnografía se propone descubrir las creencias y los valores, pero también las perspectivas y motivaciones de los individuos con relación a lo investigado, que se da especial atención a sus significados, e implica indagar para determinar cómo ocurren las cosas en su ambiente natural, y que es una orientación que puede hacer un gran aporte en la investigación educativa, al existir un creciente interés de los docentes por investigar su medio.

Pero, es importante precisar, que el uso de la etnografía no es como método, sino como metodología con " $\mathrm{m}$ " minúscula, como orientación que contribuye con el método seleccionado; y su aportación es por la facilidad que ofrece para caracterizar lo que hacen los docentes, cómo se comportan e interactúan con los alumnos mediante el uso de las TIC; además de que permite reconstruir las relaciones derivadas de los procesos educativos, esto, a través de las técnicas de recolección y análisis de datos que ofrece, como la observación, el análisis de documentos y la entrevista.

Apoya lo anterior la postura de Paradise (1994), cuando afirma que la etnografía se utiliza cada vez más para estudiar todo tipo de fenómenos sociales, por lo que parece haber sufrido cambios, que llegan a causar cierta consternación, incluso en el ámbito académico; y, agrega: Yo creo que la confusión se explica en parte por una falta de claridad en torno a la naturaleza de la etnografía como metodología con "m" minúscula, y como metodología con "M" mayúscula, para así expresarlo. Por un lado la etnografía se entiende como un conjunto de técnicas para recolectar, analizar y presentar datos (observación participante, entrevista abierta, análisis cualitativo, descripción narrativa), y por el otro lado, la etnografía se entiende más como procedimientos para la investigación socio-cultural, los cuales integran necesariamente técnicas con una perspectiva analítico-explicativa. Así que existen en la investigación educativa por lo menos dos sentidos distintos para la etnografía. (Paradise; 1994: 73, 74).
Así, la finalidad de la autora es dejar en claro cómo la etnografía, surgida de la Antropología, se ha ido desarrollando también como metodología, sobre todo, en el ámbito de la investigación educativa, pese a las múltiples críticas sufridas, por lo que concluye con la siguiente recomendación:

Que cuando hablemos de la etnografía hagamos una clara distinción entre metodología con " $\mathrm{m}$ " minúscula, ese conjunto de técnicas que se pueden comparar o contrastar con cualquier otra serie de procedimiento, y metodología con "M" mayúscula, esa perspectiva holística, sociocultural e interpretativa que integra inextrincablemente técnica con teoría. (Paradise; 1994: 80).

\section{El alcance del estudio}

"Las investigaciones por su alcance pueden ser exploratorias, descriptivas, correlacionales o explicativas, teniendo un propósito y un valor determinados según sea el caso" (Hernández; 2010: 78).

Para Hernández (2010), una vez que el investigador revisa la literatura existente sobre el tema estudiado, y resuelve que es factible la investigación, el siguiente paso es determinar su alcance, existiendo cuatro posibilidades que no deben ser consideradas como tipos, sino como un continuo de causalidad. En primer lugar están los estudios exploratorios, que se realizan cuando se trata de un problema poco estudiado, se indaga desde una perspectiva innovadora, y son trabajos que ayudan a identificar conceptos promisorios para otras investigaciones; en segundo lugar se encuentran los estudios descriptivos, que buscan especificar propiedades y características de un fenómeno, además de describir tendencias de una población; en tercer lugar se ubican los estudios correlacionales, donde se asocia variables a través de un patrón predecible para una población, ofreciendo una predicción; y, en cuarto lugar están los estudios explicativos, donde la finalidad es establecer las causas de los fenómenos y generar un sentido de entendimiento. 
Ahora bien, de acuerdo con las posibilidades presentadas, el alcance del estudio es el explicativo, ya que el propósito está dirigido a describir cómo son las prácticas de los docentes respecto al uso de las TIC; realizado lo anterior, responder por las causas de esas prácticas, explicar por qué ocurren de una manera y no de otra, esto es lo que otorgará la posibilidad de conocer si existe un desfase entre lo propuesto por una institución determinada y lo que sucede en la realidad, para estar en condiciones, finalmente, de realizar una propuesta que contribuya a mejorar el uso de las tecnologías de manera sustancial. Esto implica ir más allá de la descripción del fenómeno, para llegar a las causas que lo originan e intervenirlas.

\section{Las técnicas de recolección de datos}

"Las técnicas son medios auxiliares que concurren a la misma finalidad (Asti Vera; 1972, tomado de Bisquerra; 1989: 55). Las técnicas son particulares, mientras que el método es general. Dentro de un método pueden utilizarse diversas técnicas" (Bisquerra; 1989: 55).

La importancia de las técnicas como medios auxiliares que concurren a un mismo fin, radica en que gracias a ellas el investigador puede conocer y obtener los datos finos a partir de los cuales se podrá interpretar el fenómeno estudiado; la elección de esas técnicas está orientada principalmente por el objetivo pretendido, luego por el paradigma, la perspectiva, el método, la orientación y los alcances del estudios, pero además, por las posibilidades del sujeto que investiga para acceder al espacio natural en que suceden los hechos. En el caso concreto, las técnicas propuestas son las siguientes:

\section{La observación participante}

Se trata de una de las técnicas más importantes de la orientación etnográfica que, de acuerdo con Casanova (1998), consiste en el examen que una persona puede realizar sobre otras, o sobre algunos objetos o hechos, con la finalidad de llegar a obtener un conocimiento profundo de ellos. Así, la observación configura una especie de proceso cuya primera función es la recolección de información sobre el fenómeno investigado, pero que después debe ser codificada para ser utilizada en la interpretación de los hechos.
Para Woods (1995) la observación como técnica para la recolección de datos, permite al investigador el examen sobre determinados sujetos, objetos o hechos, y puede ser de dos tipos, participante y no participante, la primera ofrece la posibilidad de observar participando, lo que implica ser parte del grupo y tener acceso a sus actividades, y por ello, a la menor distancia posible; $y$, la segunda implica un distanciamiento entre el investigador y el objeto de estudio. Según el autor, la observación requiere un ojo avizor, un oído fino y una buena memoria.

En el mismo sentido, señala Bisquerra (1989), que en la observación participante el investigador es uno más del grupo, que se dedica a las actividades que está observando, y que por lo tanto está en condiciones de formular una definición y explicación hipotética del fenómeno estudiado.

\section{Las notas de campo}

En opinión de Shagoury (2000), la notas de campo son un método usual para la recolección de datos, que si se integran con naturalidad al trabajo de investigación pueden otorgar buenos resultados, para lo cual recomienda el uso de fichas bibliográficas, la toma de notas, y reservarse un rato para registrar acontecimientos importantes; agrega que se trata de simples observaciones de lo que se ve en el lugar investigado, pero que deben incluir a quién se observa y el contexto.

Por su parte, García (1997), señala que las notas de campo, ya analizadas, son instrumentos con que cuenta el investigador para hacer evidente la actividad que estudia, entenderla $y$, en su caso, intervenirla; es un instrumento que puede ayudar a reconstruir los hechos en un registro.

\section{La entrevista}

Se trata, según Bisquerra (1989), de una conversación entre dos personas, que tiene como finalidad la obtención de información, donde el entrevistador formula preguntas sobre los objetivos planteados, y el entrevistado responde de acuerdo con información que posee sobre el tema. 
Agrega el autor, que la información es costosa de recoger y su análisis laborioso, que llevar a cabo entrevistas implica planificación y tiempo, por lo que es importante, previo a su realización, establecer lo que se pretende conocer, especificar si se trata de hechos, opiniones o actitudes y construir un protocolo de entrevista. En opinión de Woods (1995), son conversaciones o discusiones, lo que indica un proceso libre, democrático e informal, y en el que los individuos pueden manifestarse como son; agrega que la recuperación de los diálogos tiene que quedar plasmada en un registro, que las entrevistas son también un modo de descubrir las visiones de distintas personas y recoger información sobre acontecimientos o problemas, y su objetivo es descubrir lo que se encuentra en el interior del entrevistado. Los atributos del entrevistador, según el autor, deben ser la capacidad para crear un ambiente de confianza, tener curiosidad y actuar con naturalidad.

Ahora bien, por el objetivo principal que se persigue, y que es la caracterización de las prácticas de los profesores, mediante el uso de las TIC, y la información requerida para ello, que se refiere principalmente a lo que Fuentes (1990) denomina lo cotidiano, el sentido común y la conciencia colectiva, se sugiere trabajar con entrevistas estructuradas que permitan categorizar, saturar y triangular los datos obtenidos para garantizar la validez de los resultados, pero al mismo tiempo sin alejarse de la perspectiva etnometodológica, para lo cual se requiere la formulación de un guion de trabajo, que contemple, entre otros, los aspectos que a continuación se presentan y que serán las base de las preguntas a formular.

Conocimiento del modelo académico.

Percepción de la modalidad educativa.

Rol docente a partir del modelo y la modalidad.

Rol del alumno a partir del modelo y la modalidad.

Perfil de egreso de los alumnos.

Tecnologías puestas a disposición del docente.

Uso pedagógico de las herramientas tecnológicas.
Políticas y lineamientos sobre el uso de las TIC.

Uso de las herramientas tecnológicas.

Diseño y evaluación de actividades.

Contribución al perfil de egreso del alumno.

Problemas en el uso de los recursos tecnológicos.

Ventajas del uso de las tecnologías.

Desventaja del uso de las tecnologías.

Propuestas.

Necesidades.

\section{Las grabaciones}

Para Shagoury (2000), se trata de un recurso maravilloso para los investigadores, que permite la recuperación de todas las ideas expresadas por quienes interactúan en un trabajo de investigación; las grabaciones, al igual que las notas de campo, son el principal recurso para la elaboración de registros; sin embargo, señala la autora que se debe ser consciente que la grabadora es un instrumento que no puede suplir al observador. En este tipo de trabajos, las grabaciones se consideran de suma utilidad en la realización de las entrevistas, pues a partir de ellas será posible recuperar todos los datos expuestos por los entrevistados.

\section{Los registros}

En opinión de Woods (1995), se trata de la integración de información recuperada de los datos obtenidos de las notas de campo o de las grabaciones; pueden ser de dos tipos, simples, cuando se trata de la información textual que arrojan los instrumentos mencionados, en cuyo caso se ha de cuidar que no haya interpretaciones personales, que de ser necesarias, deberán acompañarse de una simbología especial; y, ampliados, cuando se hace un trabajo posterior para identificar acciones, subcategorías, categorías e incluso megacategorías que permitan construir esquemas para interpretar de forma más simple el fenómeno estudiado. 
En este caso, se propone la elaboración de un registro simple por cada entrevista formulada, para después ampliarlo mediante el análisis de las respuestas otorgadas, y estar en condiciones de describir lo que sucede en las prácticas de los docentes mediante el uso de las tecnologías.

\section{Las técnicas de análisis de datos}

"Una de las técnicas de análisis de datos más características de la metodología cualitativa es la “triangulación”. El principio básico consiste en recoger y analizar datos desde distintos ángulos para compararlos y contrastarlos entre sí" (Bisquerra; 1989: 264).

Como se asentó con antelación, son varias las técnicas para la recolección de datos dentro de la metodología cualitativa, datos que son la materia prima para la interpretación de los hechos estudiados; pero, llevar a cabo ese análisis, requiere también de una técnica, sugiriendo la triangulación, definida por Kemmis (tomado de Bisquerra 1989: 264) como:

"un control cruzado entre diferentes fuentes de datos: personas, instrumentos, documentos o la combinación de todos ellos".

La importancia de esta técnica radica en que permite conocer un mismo hecho desde diferentes ángulos, eso es lo que otorga la seguridad de que no se escapa ningún detalle a la mirada del investigador, quien podrá llegar a conclusiones inequívocas.

Para Bisquerra (1989), la triangulación ha de entenderse en sentido amplio, ya que la misma puede realizarse de cuatro maneras distintas o mediante una combinación de ellas, describiéndolas en los términos siguientes:
Triangulación de datos, consiste en la recolección de datos de distintas fuentes; puede ser temporal si los datos se recogen en distintos momentos para saber si los resultados se mantienen; espacial si los datos se toman en lugares diferentes para buscar coincidencias; y, personal si los datos provienen de diferentes personas, para contrastar resultados e identificar coincidencias; triangulación de investigadores, aquí lo que se tiene son diferentes investigadores observando un mismo fenómeno, para luego trabajar sobre las coincidencias encontradas; triangulación teórica, se refiere a la revisión de diferentes teorías, que incluso pueden ser divergentes, y la finalidad es llegar a una interpretación más amplia del fenómeno; triangulación de metodologías, tiene lugar cuando se utilizan diferentes métodos respecto del estudio de un mismo acontecimiento, con la finalidad de estudiar coincidencias y divergencias; $\mathrm{y}$, triangulación múltiple, se realiza cuando el investigador hace una combinación de dos o más de los tipos de triangulación, la idea es comparar los resultados obtenidos para encontrar las coincidencias que le permitan analizar mejor un fenómeno determinado.

Así, sin perder de vista que el objetivo es caracterizar las prácticas de los profesores respecto al uso de las TIC, donde las entrevistas tienen un papel preponderante, lo que se recomienda es la triangulación de datos, sobre todo de los que provienen de las personas involucradas en las actividades de enseñanzaaprendizaje, y que son los docentes, alumnos y el personal directivo de la institución educativa de que se trate; por ello, en un primer momento se debe hacer la saturación de la información obtenida de cada grupo de personas para definir coincidencias; después, la triangulación de los resultados generales, para nuevamente localizar convergencias y estar en condiciones de describir lo que sucede en la institución, analizarlo e interpretarlo, para conocer las causas que caracterizan las prácticas, eso otorgará elementos para triangular, en un tercer momento, con la información obtenida por otros medios, y determinar si existe alineación o no, con lo dispuesto en las políticas y lineamientos institucionales sobre el uso de las TIC; y, en su caso, poder elaborar una propuesta que contribuya al mejoramiento de la labor docente en beneficio de los alumnos. 


\section{La población y muestra}

"El investigador delimita el ámbito de su estudio definiendo una población. La población es el conjunto de todos los individuos en los que se desea estudiar el fenómeno... Sin embargo, en la práctica no se analizan todos los individuos de la población; sino que se selecciona una muestra. La muestra es un subconjunto de la población, seleccionado por algún método de muestreo, sobre el cual se realizan las observaciones y se recogen los datos" (Bisquerra; 1989: 81).

Con independencia del nivel educativo e institución de que se trate, la población se constituye por la totalidad de sus profesores, ese es el universo para el trabajo, y el objeto estudiado son las prácticas de esos docentes mediante el uso de las tecnologías de la información y la comunicación.

Ahora bien, en lo referente a la formulación de entrevistas, se debe hacer la selección de una muestra del total de los profesores que sea representativa, aspecto primordial para que las conclusiones sean generalizables.

Lo anterior implica la utilización de un método de muestreo, que según Bisquerra (1989), puede ser probabilístico, como el aleatorio simple, el aleatorio sistemático, el aleatorio estratificado o el muestreo por conglomerados; o no probabilístico, como el de cuotas, el intencional, el causal y la bola de nieve; cada uno con particularidades que pueden contribuir a la obtención de resultados confiables, dependiendo del universo de individuos que constituyen la población.

Para este caso, de recomienda que la muestra sea definida mediante el método causal, según el cual se puede utilizar a individuos con los que se tiene facilidad de acceso, procurando siempre una serie de medidas para asegurar la representatividad. Bisquerra (1989).

Así, acorde con el método causal, se puede elegir, por ejemplo, a un grupo de profesores con los cuales se tenga fácil acceso, tomando como medidas particulares, entre otras, que cuenten con una cierta experiencia docente en la institución, que hayan participado en procesos de formación docente, que impartan varias unidades de aprendizaje, y que puedan ser el canal de acceso a otros profesores.
Pero, con la finalidad de que las conclusiones que se obtengan sean válidas y aplicables a la totalidad de la planta docente, y toda vez que se trata de una investigación de corte cualitativo, será necesario hacer uso de determinados procedimientos.

Al respecto, señala Pérez (1994: 80), que la validez se refiere a la exactitud en los resultados; y, citando a Hansen (1979), señala que la validez, como tal, exige dos cuestiones fundamentales que son: "Estimación de la medida en que las conclusiones representan efectivamente la realidad empírica; $y$, estimación de si los constructos diseñados por los investigadores representan o miden categorías reales de la experiencia humana".

Como alternativa, la autora referida propone los procesos de saturación y triangulación de las subcategorías y categorías obtenidas del análisis de los datos, por lo que se recomienda que la información derivada de las entrevistas a los docentes sea triangulada a su vez, con la que se obtenga de entrevistas formuladas a alumnos y a directivos; la justificación obedece a lo siguiente:

En el caso de los alumnos, porque es en quienes se centra el trabajo de los profesores, y son quienes tienen una visión general, no sólo de los que fueron entrevistados, sino de la totalidad de los que los han acompañado en su trayectoria escolar, por lo que su punto de vista será de gran importancia; así, se debe entrevistar también a un grupo de alumnos, elegidos igualmente mediante el método causal; en este caso las medidas que se pueden tomar son: que se trate de estudiantes activos y regulares, y que estén cursando los últimso grados escolares, para que cuenten con varios años de experiencia y conozcan el trabajo de la mayoría de los maestros.

Finalmente, y también para corroborar la información de los docentes, puede entrevistar al personal directivo, que tenga a su cargo la administración de las tecnologías para el aprendizaje, la impartición de cursos de alfabetización digital tanto para los profesores como para los alumnos y la evaluación de lo que éstos realizan en sus actividades cotidianas; $y$, al igual que los estudiantes, cuentan con una visión general de lo que realiza la totalidad de los profesores en el proceso educativos. 
Finalmente, a partir de los elementos planteados, se puede contar con un marco metodológico exhaustivo, que permita la caracterización y análisis del uso de las tecnologías de la información y la comunicación en los procesos de enseñanza-aprendizaje, así como para la elaboración de una propuesta que mejore los resultados en beneficios de los estudiantes.

\section{Conclusiones}

El marco metodológico es, a grandes rasgos, el conjunto de acciones encaminadas a describir y analizar un objeto de estudio determinado; su importancia radica en que de su correcta elección dependerá dar o no una respuesta cierta a la pregunta de investigación; en este sentido, y teniendo presente que el objeto a estudiar es el uso de las TIC en la educación, donde se busca la comprensión de lo que sucede desde una perspectiva humanística y en un lugar y tiempo determinado, que permita contribuir a la mejora de los procesos de enseñanza-aprendizaje mediados por tecnologías, la propuesta particular es la siguiente:

\begin{tabular}{|l|l|}
\hline \multicolumn{2}{|c|}{ Marco metodológico } \\
\hline Paradigma. & Cualitativo. \\
\hline $\begin{array}{l}\text { Perspectiva humanístico- } \\
\text { cualitativa. }\end{array}$ & Etnometodológica. \\
\hline Método. & Inductivo. \\
\hline Orientación. & Etnográfica. \\
\hline Alcances del estudio. & Explicativo. \\
\hline $\begin{array}{l}\text { Técnicas de recolección } \\
\text { de datos. }\end{array}$ & $\begin{array}{l}\text { Observación participante, } \\
\text { notas de campo, } \\
\text { entrevistas, grabaciones y } \\
\text { registros. }\end{array}$ \\
\hline $\begin{array}{l}\text { Técnica de análisis de } \\
\text { datos. }\end{array}$ & $\begin{array}{l}\text { Categorización, saturación } \\
\text { y triangulación. }\end{array}$ \\
\hline Población y muestra. & Método causal. \\
\hline
\end{tabular}

Tabla 1 Elementos que integran el marco metodológico siguiente:

La propuesta planteada ofrece lo

El paradigma cualitativo permite recolectar datos sin medición numérica, para describir preguntas de investigación en el proceso de interpretación y realizar descripciones detalladas, partiendo de la idea que toda cultura tiene un modo de entender los fenómenos. Sus características son la exploración en profundidad y que los significados se extraen de los datos, el proceso es inductivo, no tiene secuencia lineal, y sus bondades son la amplitud y riqueza interpretativa.
La perspectiva etnometodológica facilita el abordaje de actividades prácticas y razonamientos sociológicos, además de estudiar actividades cotidianas, por ejemplo, cómo la gente da sentido a lo que hace.

El método inductivo promueve el análisis de casos particulares a partir de los cuales se extraen conclusiones generales, inicia con la recolección de datos, se categorizan las variables observadas, se establecen regularidades y se puede llegar a una generalización.

La orientación etnográfica se interesa por lo que hacen las personas, se propone descubrir sus creencias, valores, perspectivas, motivaciones y el modo en que esto se desarrolla; se apoya en técnicas de recolección de datos como la observación participante, notas de campo, entrevistas, grabaciones y registros; $y$, en técnicas de análisis de datos como la categorización, saturación y triangulación.

El alcance del estudio explicativo permite responder por las causas de los fenómenos.

Finalmente, en cuanto a la población y muestra, la primera queda constituida por los docentes y alumnos de la institución en que se lleva a cabo la investigación, y la segunda se sugiere sea definida a través del método causal, que permite llegar a los sujetos indicados y cuya información puede proporcionar mayores elementos para la caracterización de lo que en ese contexto sucede, así como para la construcción y puesta en marcha de una propuesta de intervención que ayude a innovar los procesos de enseñanza y mejorar los de aprendizaje.

\section{Referencias}

Bisquerra, Rafael (1989). "Métodos de Investigación Educativa. Guía Práctica", Editorial CEAC. Barcelona, España.

Casanova, Ma. Antonia. (1998). "Manual de Evaluación Educativa". Editorial la Muralla, Madrid, España.

Fuentes G., A. (1990). Harold Garfinkel: la etnometodología. Revista de Sociología, 0 (5). doi:10.5354/0719-529X.1990.27606 
García, Herrera Adriana Piedad. (1997). "La Instrumentación Metodológica en la Recuperación de la Práctica Docente, en la antología: Entorno a la Intervención de la Práctica Educativa." Editorial UNED, México.

Hernández, Sampieri Roberto, Carlos Fernández Collado y María del Pilar Baptista Lucio. (2010). "Metodología de la Investigación". Editado por Mc Graw Hill, quinta edición. Perú.

McMillan, James H., Sally Schumacher (2005). "Investigación educativa", quinta edición, editorial Pearson Educación, S. A., Madrid, España.

Paradise, Ruth (1994). "Etnografia: ¿técnicas o perspectiva epistemológica?". En Rueda Beltrán Mario, Delgado Ballesteros Gabriela y Jacobo Zardel (Coordinadores). "La etnografía en educación. Panorama, prácticas y problemas". Editado por CISE/UNAM, pp. 173208. México.

Pérez, Serrano Gloria. (1994). "Investigación Cualitativa, Retos e Interrogantes. Tomo II. Técnicas y Análisis de Datos". Editorial La Muralla, Madrid, España.

Shagoury, Hubbard Ruth y Brenda Miller Power. (2000). "El Arte de la Indagación en el Aula. Manual para docentes investigadores." Editorial Gedisa. Barcelona, España.

Woods, Peter. (1995). "La Escuela por Dentro. La Etnografía en la Investigación Educativa." Editorial Paidós, tercera reimpresión. España 